Artigo Original

\title{
Fatores que influenciam o prognóstico deambulatório nos diferentes níveis de lesão da mielomeningocele
}

\section{Factors they influence the prognostic ambulatory for the different levels of injury of the myelomeningocele}

\author{
Ramos FS ${ }^{1}$, Macedo LK' 1 , Scarlato $A^{2}$, Herrera $\mathrm{G}^{2}$
}

\section{RESUMO}

Objetivo: determinar quais fatores influenciam o prognóstico deambulatório nos diferentes níveis de lesão da mielomeningocele, adicionando informações relevantes para a conduta fisioterapêutica. Metodologia: revisão bibliográfica através de livros do acervo da biblioteca do Centro Universitário São Camilo e artigos científicos pesquisados pelas bases de dados Medline e Lilacs, compreendendo o período de 1966 à 2004. Discussão: níveis de lesão, alterações ortopédicas, idade, motivação familiar, déficit de equilíbrio, alterações cognitivas, tratamento fisioterapêutico, dentre outros fatores, são citados na literatura como positivos ou negativos no prognóstico deambulatório dos pacientes com mielomeningocele; embora nenhum artigo tenha citados todos os fatores. Conclusão: há consenso na literatura sobre a influência dos níveis de lesão e das alterações ortopédicas sobre a deambulação. Embora havendo discordância na literatura, os demais fatores são de suma importância no estabelecimento do prognóstico deambulatório para os pacientes com mielomeningocele.

Unitermos: Mielomeningocele, Espinha Bífida, Fisioterapia, Marcha.

Citação: Ramos FS, Macedo LK, Scarlato A, Herrera G. Fatores que influenciam o prognóstico deambulatório nos diferentes níveis de lesão da mielomeningocele. Rev Neurociencias 2005; 13(2):080-086.

\section{SUMMARY}

Objective: to determine which factors they influence the prognostic ambulatory for the different levels of injury of the MMC, adding excellent information for the manaje physical therapy. Method: bibliographical revision through books that are part of the quantity of the Padre Innocente Radrizzani library of the Centro Universitário São Camilo and scientific articles searched by the databases Medline and Lilacs, understanding the period of 1966 to the 2004. Discussion: levels of injury, ortophaedics alterations, age, familiar motivation, socialization, deficit of balance, cognitivas alterations, physical therapy, energy expense, amongst other factors, are cited in literature as positive or negative in the prognostic ambulatory of the patients with MMC; although no article has cited all the factors. Conclusion: it has consensus in literature on the influence of the levels of injury and the orthopaedics alterations on the ambulation. Although having discord in literature, the too much factors are of utmost importance in the establishment of the prognostic ambulatory for the patients with MMC.

Keywords: Myelomeningocele, Spina Bifida, Physical Therapy, Gait.

Citation: Ramos FS, Macedo LK, Scarlato A, Herrera G. Factors they influence the prognostic ambulatory for the different levels of injury of the myelomeningocele. Rev Neurociencias 2005; 13(2):080-086.

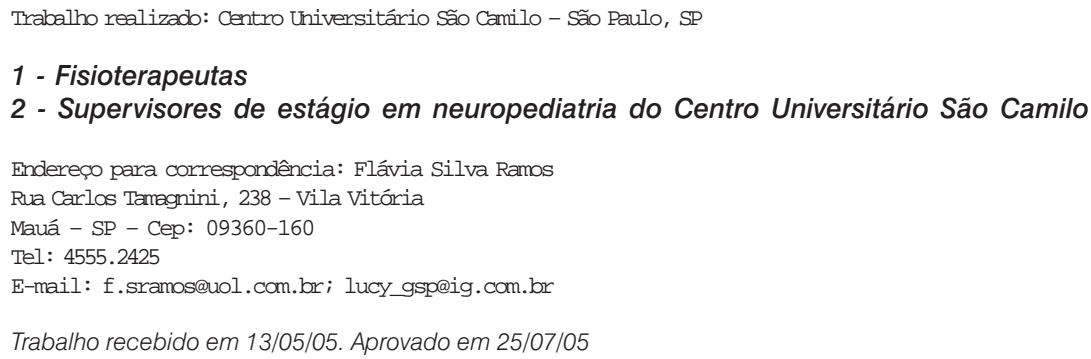




\section{INTRODUÇÃO}

Mielomeningocele é uma malformação congênita do Sistema Nervoso Central caracterizada por falta de fusão dos arcos vertebrais posteriores. Através dessa falha ocorre a extrusão de tecido nervoso, formando um cisto na coluna vertebral. Esse cisto é composto por medula espinal, raízes nervosas, meninges e preenchido por líquido cefalorraquidiano, sendo recoberto ou não por pele ${ }^{1,2}$.

O resultado deambulatório dos pacientes com mielomeningocele na adolescência e vida adulta recebeu mais atenção conforme os pacientes foram beneficiados pelo tratamento da hidrocefalia ${ }^{3}$ e pela intervenção cirúrgica intra-útero ${ }^{4}$.

Segundo alguns autores ${ }^{5}$, a deambulação pode ser afetada por diversos fatores nesses indivíduos. O termo "deambulação" indica locomoção em posição ereta com ou sem dispositivos auxiliares. É possível determinar o potencial para a marcha em crianças portadoras de espinha bífida, desde que se conheça os fatores que influenciam o prognóstico em relação à marcha ${ }^{6}$.

Esse trabalho teve como objetivo determinar quais fatores influenciam o prognóstico deambulatório para os diferentes níveis de lesão da mielomeningocele, adicionando informações relevantes para a conduta fisioterapêutica.

\section{METODOLOGIA}

Revisão bibliográfica através dos principais livros referentes ao tema e artigos científicos pesquisados pelas bases de dados Medline e Lilacs, compreendendo o período de 1966 à 2004, através das palavras-chave: mielomeningocele, espinha bífida, fisioterapia, marcha, deambulação. Foram incluídos artigos de revisão bibliográfica e pesquisas de campo; e excluídos os relatos de caso.

\section{RESULTADOS}

Na revisão bibliográfica desse estudo foram encontrados 20 artigos sobre os fatores que podem influenciar a deambulação na mielomeningocele, sendo que alguns artigos citaram mais de um fator, conforme Gráfico 1.

\section{DISCUSSÃO}

Tendo em vista a citação dos níveis de lesão neurológica em $40 \%$ dos artigos encontrados, foi con-

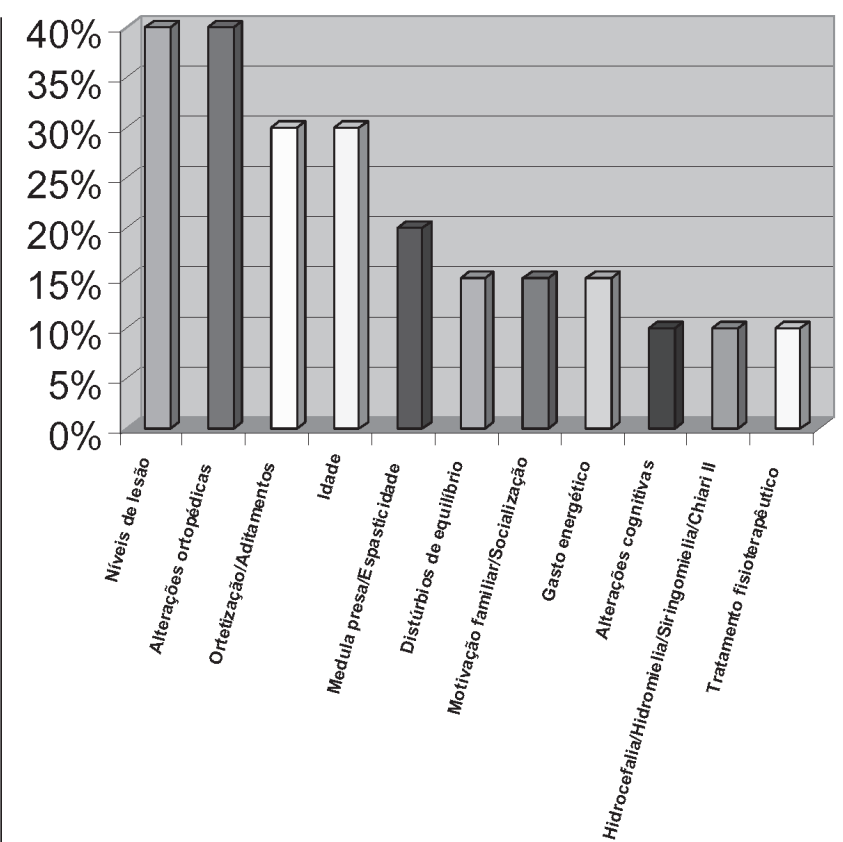

Gráfico 1. Representação dos fatores de influência sobre a deambulação na Mielomeningocele encontrados na literatura de acordo com sua incidência.

siderado um importante fator prognóstico da deambulação na mielomeningocele.

Parece haver concordância na literatura sobre a influência do nível de lesão neurológica em relação ao prognóstico deambulatório na mielomeningocele. Porém, nos estudos encontrados não houve consenso sobre a classificação dos níveis de lesão utilizada. A classificação em quatro níveis de lesão (nível torácico, nível lombar alto, nível lombar baixo e nível sacral) criada por Hoffer et al. ${ }^{7}$ foi a mais utilizada entre os estudos analisados ${ }^{8-10}$. Outra classificação empregada foi a de Sharrard (1964) ${ }^{11}$ em três níveis de lesão (nível torácico, nível lombar e nível sacral), esta no estudo de Bartonek et al. ${ }^{11}$. Nos outros estudos ${ }^{5,12}$ não foi citada a classificação utilizada.

É relevante o estudo de Bartonek et al. ${ }^{9}$ onde há a comparação entre seis sistemas de classificação do nível de lesão neurológica, sendo os de Sharrard (nível torácico, nível L1, nível L2, nível L3, nível L4, nível L5, nível S1 e nível S2), Hoffer et al. ${ }^{7}$ (nível torácico, nível lombar alto, nível lombar baixo e nível sacral), Lindseth 1976 (nível torácico, nível L1, nível L2, nível L3, nível L4, nível L5 e nível sacral), Broughton et al. 1993 (nível torácico, nível L1, nível L2, nível L3, nível L4, nível L5, nível S1 e nível S2), Ferrari et al. 1985 (nível torácico, nível L1, nível L2, nível L3, nível L4, nível L5, nível S1, nível S2 e nível S3) e McDonald et al. 1991 (nível 
L1, nível L3 e nível sacral). Esse estudo sugere que comparações de resultados de tratamentos são difíceis e confusas devido aos diversos sistemas de classificação usados para descrever a função motora e o nível neurológico da lesão nos portadores de mielomeningocele.

O prognóstico deambulatório de acordo com os níveis de lesão neurológica mais citado na literatura aparentemente é o de Hoffer et al. ${ }^{7}$, no qual dos pacientes com lesão no nível torácico espera-se a ausência de deambulação; dos pacientes com nível lombar a deambulação comunitária, a domiciliar ou a não-funcional de acordo com outros fatores de influência (como retardo mental, deformidades ortopédicas, idade, fraturas e espasticidade); e dos pacientes com nível sacral espera-se a deambulação comunitária.

Bartonel et al. ${ }^{11}$, apesar de utilizarem outra classificação (Sharrard), alcançaram resultados equivalentes à pesquisa de Hoffer et al. ${ }^{7}$. Entretanto, Charney et al. ${ }^{5}$ verificaram que sujeitos com paralisia em nível alto (torácico e lombar alto) poderiam alcançar a deambulação comunitária, porém nesse estudo não houve distinção entre os níveis de lesão, não foi citada a classificação utilizada e somente foram relatados três fatores (retardo mental, fisioterapia e conformidade dos pais) que poderiam afetar o prognóstico deambulatório.

Assim como os níveis de lesão, as alterações ortopédicas foram encontradas em $40 \%$ dos artigos analisados. Isso sugere que as alterações ortopédicas têm peso similar ao nível de lesão no prognóstico deambulatório na mielomeningocele.

Em $75 \%$ desses estudos as alterações ortopédicas foram citadas como um fator que influencia negativamente o prognóstico deambulatório. Essas alterações ortopédicas incluem: deformidades da coluna ${ }^{7,8,10}$, aumento da torsão tibial externa ${ }^{13}$, deformidade em flexão do joelho ${ }^{14}$ e deformidade em flexão do quadril| ${ }^{8}$.

Em 25\% desses estudos as alterações ortopédicas foram citadas como um fator indiferente ao prognóstico deambulatório. As alterações ortopédicas citadas foram: luxação do quadril11,15 e aumento da obliqüidade pélvica ${ }^{8}$.

É importante ressaltar que esses estudos envolveram sujeitos com níveis de lesão e idades diferentes. Outro aspecto de relevância é a intervenção cirúrgica nas alterações ortopédicas como um fator positivo no prognóstico deambulatório, como foi citado em 1973 por Hoffer et al. ${ }^{7}$ e em 1992 por Swank et al. ${ }^{12}$.
Em relação à ortetização e uso de aditamentos, esses fatores foram citados em $30 \%$ dos artigos analisados. Foi relatado que alguns fatores interferem no resultado da ortetização e utilização de aditamentos e podem, conseqüentemente, afetar o prognóstico deambulatório na mielomeningocele.

Gerritsma-Blecker et al. ${ }^{16}$, apesar de não terem citado o nível de lesão estudado, encontraram que, apesar das contra-indicações de alguns projetos ortésicos, quando realizada a indicação adequada da órtese essa tem efeito positivo na deambulação nos casos de mielomeningocele. Thomas et al. ${ }^{17}$ também avaliaram a eficiência da ortetização (órteses tornozelo-pé) sobre o padrão de marcha nos portadores de mielomeningocele (níveis lombar baixo e sacral) e concluíram que a utilização dessas órteses mantém e melhora os parâmetros básicos da marcha, aumentando a mobilidade; entretanto esses autores reconheceram a amostra pequena do estudo $(n=30)$.

Katz et al. ${ }^{18} \mathrm{em}$ estudo sobre dois projetos ortésicos para indivíduos com nível torácico e lombar alto de mielomeningocele, apesar da amostra pouco significativa $(n=8)$, puderam concluir que é importante a avaliação adequada dos pacientes em relação aos gastos funcionais e energéticos e ao custo financeiro de cada projeto. Cuddeford et al. ${ }^{19}$ também avaliaram o gasto energético na deambulação com órteses, o estudo contou com 26 sujeitos (nível de lesão entre T12-L4) e concluíram que há muitos fatores a serem considerados na prescrição ortésica, além do gasto energético e que essa intervenção deve ser adequada individualmente aos portadores de mielomeningocele.

Vankoski et al. ${ }^{3}$ realizaram estudo com objetivo de verificar o efeito das muletas canadenses no padrão de marcha das crianças com mielomeningocele. Foi conclusão dos autores que os desvios na biomecânica da pelve e do quadril são relacionados à fraqueza muscular dessa região e melhoram com o uso de muletas canadenses; as muletas permitem que o paciente distribua o peso em seus membros superiores e reduza o trabalho da fraca musculatura dos membros inferiores, permitindo que o paciente mantenha um padrão de marcha funcional próximo ao normal.

Foi encontrado por Mazur et al. ${ }^{20}$ que nos níveis torácico e lombar alto de paralisia a ortetização e o treino de marcha são controversos na literatura, enquanto no nível lombar baixo essa proposta terapêutica é eficiente na melhora do prognóstico deambulatório. 
Em relação à idade, esse fator foi citado em $30 \%$ dos estudos analisados, sendo considerado de influência sobre a deambulação em $66 \%$ desses artigos $5,7,8,21$.

O estudo de Hoffer et al. ${ }^{7}$ citou a idade como um fator importante no prognóstico deambulatório dos pacientes com nível de lesão lombar. Todos os pacientes com esse nível que conseguiram a deambulação funcional o fizeram antes dos 9 anos de idade e já possuíam o status de deambuladores não-funcionais aos 5 anos de idade. Sendo a idade indiferente no prognóstico deambulatório dos sujeitos com outros níveis de lesão.

No estudo de Samuelsson et al. ${ }^{8}$ com 163 pacientes (idade média 14 anos e 10 meses e todos os níveis de lesão), a idade teve poder discriminativo sobre a deambulação nos pacientes entre 6 a 16 anos (os deambuladores comunitários tinham idade média de 15 anos e 10 meses, os deambuladores domiciliares 17 anos e 4 meses e os cadeirantes 13 anos e 5 meses). Não houve relação entre a idade e os outros níveis de lesão nesse estudo.

Outro estudo é o de Charney et al..$^{5}$ (87 sujeitos com 5 anos de idade), que analisou se crianças com nível alto de paralisia conseguiriam a deambulação e se isso fosse positivo, por quanto tempo a deambulação poderia ser mantida. Cinqüenta e dois por cento dos pacientes conseguiram a deambulação comunitária aos 5 anos, 45\% não conseguiram aos 5 anos nem depois (12\% sendo deambuladores domiciliares e $7 \%$ cadeirantes). Os autores associaram outros fatores (retardo mental e ausência de tratamento fisioterapêutico) na explicação desses resultados e ainda citaram que embora a maioria (60\%) dos deambuladores comunitários mantivesse a marcha na adolescência, começou-se a ver a tendência previamente relatada de que somente uma minoria dos indivíduos com nível alto de paralisia continuaria a deambular na vida adulta.

No estudo de Williams et al. ${ }^{21}(n=173)$ foi constatado que existe um atraso na realização da deambulação para todos os níveis de lesão neurológica, entretanto os autores não explicaram o motivo desse atraso. Além disso, a idade de cessação da deambulação em pacientes com paralisia alta pode ser muito mais precoce (entre 6-9 anos) do que o previamente relatado na literatura, tendo como razões o período de rápido crescimento, ganho de peso no início da puberdade e a perda neurológica. Em longo prazo, a idade também parece ser um fator de influência na perda da deambulação, como percebido nos resultados do estudo supra citado (ao final do estudo: 2 crianças com lesão torácica ainda deambulavam com a idade média de 8 anos \pm 1 mês; 3 com lesão lombar alta com a idade média de 12 anos \pm 3 anos; 5 com lesão lombar média com a idade média de 8 anos e 6 meses \pm 3 anos e 4 meses; 28 com lesão lombar baixa com idade média de 8 anos e 8 meses \pm 4 anos; 65 com lesão sacral com a idade média de 9 anos \pm 5 anos). Nesse estudo fica clara a relevância da idade como fator significante para o prognóstico deambulatório, principalmente quando esta é associada com o nível de lesão neurológica.

A idade foi considerada como um fator indiferente ao prognóstico deambulatório em 33\% dos artigos que citaram esse fator ${ }^{10,15}$.

No estudo de Norrlin et al. ${ }^{10}$ a idade não foi relacionada com a mobilidade, embora os autores esperassem por essa relação. No estudo de Gabrieli et al. ${ }^{15}$ não houve diferença estatística em relação à idade entre os dois grupos (grupo 1, pacientes que não apresentavam luxação do quadril ou que apresentavam luxação simétrica; grupo 2, pacientes que apresentavam luxação assimétrica do quadril). Em ambos os estudos, a falta de relacionamento entre a idade e o prognóstico deambulatório não foi explicado pelos autores.

Sobre a influência da síndrome da medula presa e da espasticidade na deambulação, esses fatores foram incluídos em $20 \%$ dos artigos analisados.

No estudo de Swank et al..$^{12}$ a presença de medula presa foi o fator primário para a perda significativa da função motora. Dos 206 sujeitos, 11 tiveram perda significativa do nível funcional entre a avaliação inicial no nascimento e a última avaliação do estudo e todos esses pacientes eram portadores de medula presa.

Hoffer et al. ${ }^{7}$ citou a espasticidade como influência negativa na deambulação, juntamente com outros fatores como deformidades da coluna e fraturas. Esses autores defenderam que a redução da deambulação entre as idades de 9 a 17 anos é influenciada por esses fatores.

A espasticidade também foi um fator de influência em relação à deambulação no estudo de Samuelsson et al. ${ }^{8}$. Em quase metade dos sujeitos com nível de lesão abaixo de L1-L2, a espasticidade, em associação com a siringohidromielia e malformação de Chiari tipo II, influenciou negativamente a habilidade da marcha.

No estudo de Bartonek et al. ${ }^{11}$ a espasticidade era presente em todos os níveis funcionais exceto o I 
(nível de lesão S2) e foi considerada um fator significativo para não se alcançar a deambulação esperada. Embora sem significado estatístico, a espasticidade em associação com contraturas em flexão do quadril ou do joelho pareceu também influenciar o prognóstico deambulatório negativamente.

Em relação aos distúrbios de equilíbrio e sua influência no prognóstico deambulatório, houve citação em 15\% dos artigos analisados.

No estudo de Swank et al. ${ }^{22}$, foi realizado prognóstico deambulatório de acordo com o nível da lesão neurológica associado com o equilíbrio em sedestação. Os autores encontraram que as variáveis nível motor e equilíbrio em sedestação predisseram corretamente $89 \%$ do status deambulatório dos 26 sujeitos desse estudo. Os dados sugeriram que somente três grupos de pacientes têm probabilidade de serem deambuladores: pacientes com nível lombar ou sacral sem déficit de equilíbrio em sedestação e os pacientes com nível sacral e com déficit médio de equilíbrio em sedestação; de todas as outras combinações, espera-se a ausência de deambulação.

Em 1992, Swank et al. ${ }^{12}$ já haviam realizado um estudo com resultado semelhante. Dos 206 sujeitos, 30 tinham leve déficit de equilíbrio, 29 tinham moderado e 16 tinham déficit severo; 48 meses após o estudo, nenhum dos pacientes com déficit moderado ou severo eram deambuladores comunitários e todos os pacientes com déficit severo eram não-deambuladores.

Outro estudo é o de Bartonek et al. ${ }^{11}$ onde foram estabelecidas diferenças significativas entre o grupo que alcançou a deambulação prevista e o grupo que não alcançou, com base do déficit de equilíbrio durante a marcha, a sedestação e o ortostatismo. O número de sujeitos com déficit de equilíbrio nesse estudo foi significativamente maior entre aqueles que tiveram duas ou mais revisões da derivação ventricular.

A motivação familiar e a socialização foram citadas como fatores de influência na deambulação em 15\% dos artigos.

Embora sem detalhes, os fatores sociais foram citados no estudo de Hoffer et al. ${ }^{7}$ como influentes no prognóstico deambulatório.

No estudo de Norrlin et al. ${ }^{10}$ foi relatado que a atitude de apoio dos pais, assim como a adaptação ambiental, pode ser insuficiente na motivação da mobilidade em crianças mais velhas, embora isso não tenha sido confirmado pelos autores. Isso é contrário ao relatado no estudo de Charney et al. ${ }^{5}$, onde os autores encontraram que dos 45 sujeitos que conseguiram a deambulação, $68 \%$ tinham pais colaborativos; com a conformidade dos pais nas atividades de marcha, mesmo sendo um aspecto de difícil avaliação sistemática, um fator significativo nesse estudo, que tinha como objetivo determinar quais fatores ou variáveis afetariam a deambulação comunitária em pacientes com nível alto de paralisia.

O gasto energético como fator de influência no prognóstico deambulatório foi citado em 15\% dos artigos.

Como citado anteriormente, segundo Katz et al. ${ }^{18}$ é importante a avaliação adequada dos pacientes em relação aos gastos funcionais e energéticos e ao custo financeiro de cada projeto. Conforme também já citado, Cuddeford et al. ${ }^{19}$ tiveram como conclusão que há muitos fatores a serem considerados na prescrição ortésica, incluindo o gasto energético e que essa intervenção deve ser adequada individualmente aos portadores de mielomeningocele.

A influência das alterações cognitivas no prognóstico deambulatório foi citada em 10\% dos artigos encontrados.

A alteração cognitiva (retardo mental) foi encontrada no estudo de Charney et al. ${ }^{5}$ como um fator de influência negativa no prognóstico deambulatório dos portadores de mielomeningocele, embora esse fator isoladamente não impedisse a deambulação. Nesse estudo com 87 pacientes, um esquema multivariado identificou três fatores mais importantes que afetam a deambulação, sendo eles o retardo mental, a ausência de acompanhamento fisioterapêutico e a falta de colaboração familiar.

A presença de sintomas severos de disfunção cerebral foi citada por Norrlin et al..$^{10}$ como relevante na quantificação do auxílio necessário para portadores de mielomeningocele, exceto no nível sacral, tendo implicações no programa terapêutico desses indivíduos.

As alterações como hidrocefalia, hidromielia, siringomielia e malformação de Chiari II afetando o prognóstico deambulatório foram encontradas em somente $10 \%$ dos artigos analisados.

Em relação à siringomielia e malformação de Chiari II, essas alterações foram citadas no estudo de Samuelsson et al. ${ }^{8}$ como importantes fatores de causa de disfunção dos membros superiores, podendo afetar a habilidade da marcha nos níveis L1L2, contribuindo à perda da função neurológica e 
deteriorando a habilidade de deambular longas distâncias. A influência da siringomielia e malformação de Chiari II nos outros níveis de lesão não foi encontrada nesse ou nos outros artigos selecionados para essa pesquisa.

A hidrocefalia e a hidromielia foram relatadas como fatores de influência negativa sobre o equilíbrio dos portadores de mielomeningocele no estudo de Swank et al. ${ }^{12}$; dos 206 sujeitos, 196 eram portadores de hidrocefalia e 5 dos 33 pacientes que desenvolveram medula presa, eram portadores de hidromielia. Embora citados como fatores de influência, não é claro nesse estudo a relação entre a hidrocefalia e a hidromielia com o prognóstico deambulatório.

Em relação ao tratamento fisioterapêutico, este foi encontrado como um fator de influência na deambulação em 10\% dos artigos selecionados.

Sobre a conduta fisioterapêutica direcionada ao treino de marcha, Charney et al. ${ }^{5}$ a afirmaram como um fator de influência positivo no prognóstico deambulatório dos portadores de mielomeningocele. Nesse estudo com 87 pacientes, um esquema multivariado identificou três fatores mais importantes que afetam positiva ou negativamente a deambulação, sendo eles o retardo mental, o tratamento fisioterapêutico e a colaboração familiar. Dos 87 sujeitos, $89 \%$ receberam tratamento fisioterapêutico e eram deambuladores comunitários, sendo a fisioterapia relevante positivamente no prognóstico deambulatório.

No estudo de Mazur et al. ${ }^{20}$, como mencionado anteriormente, foi encontrado que nos níveis torácico e lombar alto de paralisia a ortetização e o treino de marcha são controversos na literatura, enquanto no nível lombar baixo essa proposta terapêutica é eficiente na melhora do prognóstico deambulatório.

\section{CONCLUSÕES}

Há consenso na literatura sobre a influência dos níveis de lesão e das alterações ortopédicas no que se refere a deformidades da coluna, aumento da torsão tibial externa, deformidade em flexão do joeIho e deformidade em flexão do quadril, sobre o prognóstico deambulatório na mielomeningocele. Embora havendo discordâncias na literatura, a consideração dos demais fatores (idade, medula presa, espasticidade, distúrbios de equilíbrio, motivação familiar, socialização, gasto energético, alterações cognitivas, hidrocefalia, hidromielia, siringomielia, malformação de Chiari II e tratamento fisioterapêutico) é de suma importância no estabelecimento do prognóstico deambulatório para os pacientes com mielomeningocele.

Podemos notar que os fatores facilmente visíveis, tais como as alterações ortopédicas e os níveis de lesão, receberam mais atenção; já os fatores pouco visíveis, tais como a motivação familiar e a socialização, foram abolidos em muitos estudos.

O tratamento fisioterapêutico nos pacientes portadores de mielomeningocele foi um assunto discreto nas pesquisas, porém de grande importância, sendo notável a ausência de interação e publicação de estudos científicos desses profissionais. Isso poderia ocasionar falhas na prescrição e aplicação do tratamento fisioterapêutico, prejudicando o alcance e a manutenção da deambulação nos pacientes portadores de mielomeningocele.

\section{REFERÊNCIAS BIBLIOGRÁFICAS}

1. Wentz E. Mielomeningocele. In: Kudo AN (coord.). Fisioterapia, Fonoaudiologia e Terapia Ocupacional em Pediatria. $2^{\text {nd }}$ ed. São Paulo: Sarvier, 1997.

2. Ratliffe KT. Fisioterapia Clínica Pediátrica - Guia para equipe de fisioterapeutas. São Paulo: Santos, 2000.

3. Vankoski S, Moore C, Statler KD et al. The influence of forearm crutches on pelvic and hip kinematics in children with myelomeningocele: don't throwaway the crutches. Dev Med Child Neurol 1997; 39:614-619.

4. Sbragia L, Machado IN, Rojas CEB et al. Evolução de 58 fetos com meningomielocele e o potencial de reparo intraútero. Arq Neuropsiquiatr 2004; 62(2-B):487-491.
5. Charney EB, Melchionni JB, Smith DR. Community ambulation by children with myelomeningocele and high-level paralysis. J Pediatr Orthop 1991; 11:579-582.

6. Burns YR, MacDonald J. Fisioterapia e Crescimento na Infância. São Paulo: Santos; 1999.

7. Hoffer MM, Feiwell E, Perry R et al. Functional ambulation in patients with myelomeningocele. J Bon Joint Sur 1973; 55-A(1).

8. Samuelsson L, Skoog M. Ambulation in Patients with Myelomeningocele: A Multivariate Statistical Analysis. J Pediatr Orthop 1988; 8:569-575. 
9. Bartonek A, Saraste H, Knutson LM. Comparison of different systems to classify the neurological level of lesion in patients with myelomeningocele. Dev Med Child Neurol 1999; 41:796-805.

10. Norrlin S, Strinnholm M, Carlsson M et al. Factors of Significance for Mobility in Children with Myelomeningocele. Acta Paediatr 2003; 92:204-210.

11. Bartonek A, Saraste H. Factors influencing ambulation in myelomeningocele: a cross-sectional study. Dev Med Child Neurol 2001; 43:253-260.

12. Swank M, Dias L. Myelomeningocele: A Review of Aspects of 206 Patients Treated from Birth with No Selection Criteria. Dev Med Child Neurol 1992; 34:1047-1052.

13. Lim R, Dias L, Vankoski S et al. Valgus Knee Stress in Lumbosacral Myelomeningocele: A Gait-Analysis Evaluation. J Pediatr Orthop 1998; 18:428-433.

14. Williams JJ, Graham GP, Dunne KB et al. Late Knee Problems in Myelomeningocele. J Pediatr Orthop 1993; 13:701-703

15. Gabrieli APT, Vankoski S, Dias LS, et al. Análise laboratorial de marcha na mielomeningocele de nível lombar baixo e instabilidade unilateral do quadril. Acta Ortop Bras 2004; 12(2).

16. Gerritsma-Blecker CLE, Heeg M, Vos-Niël H. Ambulation with the reciprocal-gait orthosis. Acta Orthop Scand 1997; 68(5):470-473.

17. Thomas SE, Mazur JM, Child ME et al. Quantitative Evaluation of AFO Use with Myelomeningocele Children. Z Kinderchir 1989; 44 Suppl I.

18. Katz DE, Haideri N, Song K et al. Comparative Study of Conventional Hip-Knee-Ankle-Foot Orthoses Versus Reciprocating-Gait Orthoses for Children with High-Level Paraparesis. J Pediatr Orthop 1997; 17:377-386.

19. Cuddeford TJ, Freeling RP, Thomas SS et al. Energy Consumption in Children with Myelomeningocele: A Comparison Betwenn Reciprocating Gait Orthosis and Hip-anklefoot Orthosis Ambulators. Dev Med Child Neurol 1997; 39:239-242.

20. Mazur JM, Kyle S. Efficacy of brancing the lower limbs and ambulation training in children with myelomeningocele. Dev Med Child Neurol 2004; 46:352-356.

21. Williams EN, Broughton NS, Menelaus MB. Age-related walking in children with spina bifida. Dev Med Child Neurol 1999; 41:446-449.

22. Swank M, Dias L. Walking Ability in Spina Bifida Patients: A Model for Predicting Future Ambulatory Status Based on Sitting Balance and Motor Level. J Pediatr Orthop 1994; 14:715-718. 